\title{
Enquête entomologique dans la région des savanes (République du Togo)
}

\author{
par J. ITARD
}

\begin{abstract}
RÉSUMÉ
La répartizion des glossines, dans la région des savanes, se caractérise par : 1. - Absence de Gl. morsitans, toul au moins dans l'étendue des zones prospectées.

2. - Présence de Gl. tachinoides et de Gl. palpalis gambiensis, qui coexistent fréquemment.

Ces deux espèces se rencontrent dans une végétation de type galerie, mais fixées à proximité de points d'eau permanents. GI. tachinoides est l'espèce dominante.

Du fait de l'absence de Gl. morsitans, l'élevage des bovins est possible dans les savanes comprises entre les cours d'eau.

On donne d'autre part une liste des espèces de Tabonidae capturés au cours de cetfe enquête.
\end{abstract}

Située à l'extrémité nord du Togo, la région des Savanes déborde légèrement les parallèles $10^{\circ}$ et 110 de latitude Nord, et se situe entre le méridien de Greenwich et le mérıdien $1^{\circ}$ Est (carte l).

La région des Savanes peut être divisée, du Nord au Sud, en trois secteurs: (carte l).

- L'extrémité méridionale du plateau voltaïque, accupant le Nord de la circonscription de Dapango et limité par une ligne Ponio, Namoudjoga, Dapango, Tami. II a un relıef plus ou moins accentué.

- Un massif de collines gréseuses avec des falaises abrupies, entre Dapango et Barkoissi. De ce massif partent, vers l'Ovest, la Koulou'gona, affluent de la Biankouri (frontière avec le Ghana) ; à l'Est et au Sud des affluents de la Sansargou et de la Sangabré, tributaires de l'Oti.

- Le bassin du cours moyen de l'Otı, qui coupe l'Est et le Sud de la région des Savanes, en diagonale, de Mandouri au-delà de SansannéMango. A ce secteur se rattache le bassin de la Koumangou, affluent rive gauche de l'Oti.

Climat (carte II).

La région des Savanes est incluse entre les Isohyètes $1.000 \mathrm{~mm}$ et $1.100 \mathrm{~mm}$. Les chutes de pluies s'étendent d'avril à actobre, avec un maximum en août.

La température moyenne mensuelle passe par deuxmaxima $\left(33^{\circ} \mathrm{C}\right.$ et $35^{\circ} \mathrm{C}$ ) en mars et novembre, et deux minima $\left(31^{\circ} \mathrm{C}\right.$ et $\left.27^{\circ} \mathrm{C}\right)$ en janvier et août.

L'hygrométrie ne dépasse pas 73 p. 100 en aô̂t $i$ en saison sèche elle s'abaisse jusqu'à 15 p. $100 \mathrm{du}$ fait de l'harmattan. Ce vent desséchant souffle du Nord durant deux à trois mois à partir de fin décembre.

Hydrologie.

Seuls l'Oti, la Koumangou et quelques-uns de leurs principaux affluents peuvent être considérés 


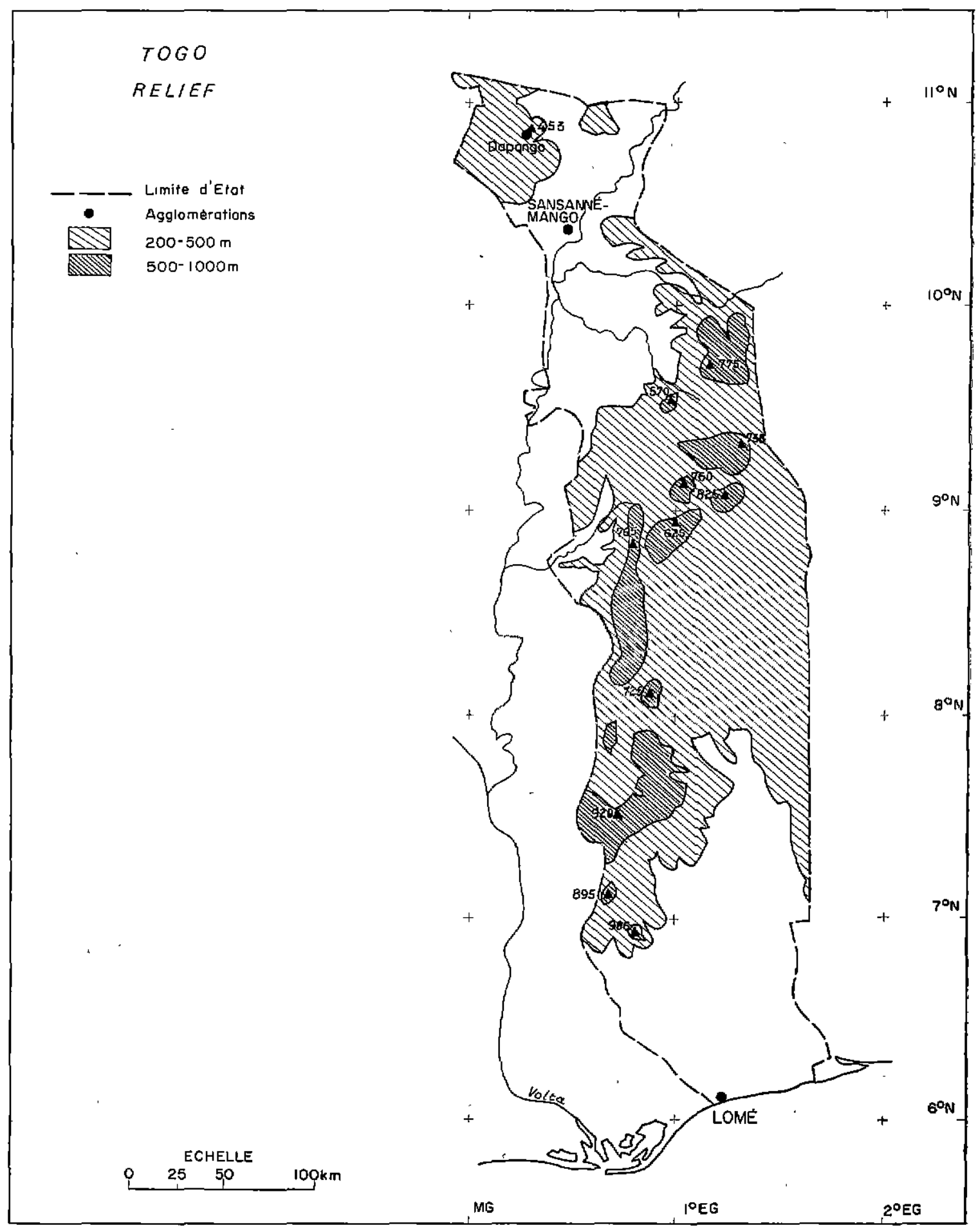

Carte I. — République du Togo — Relief. 


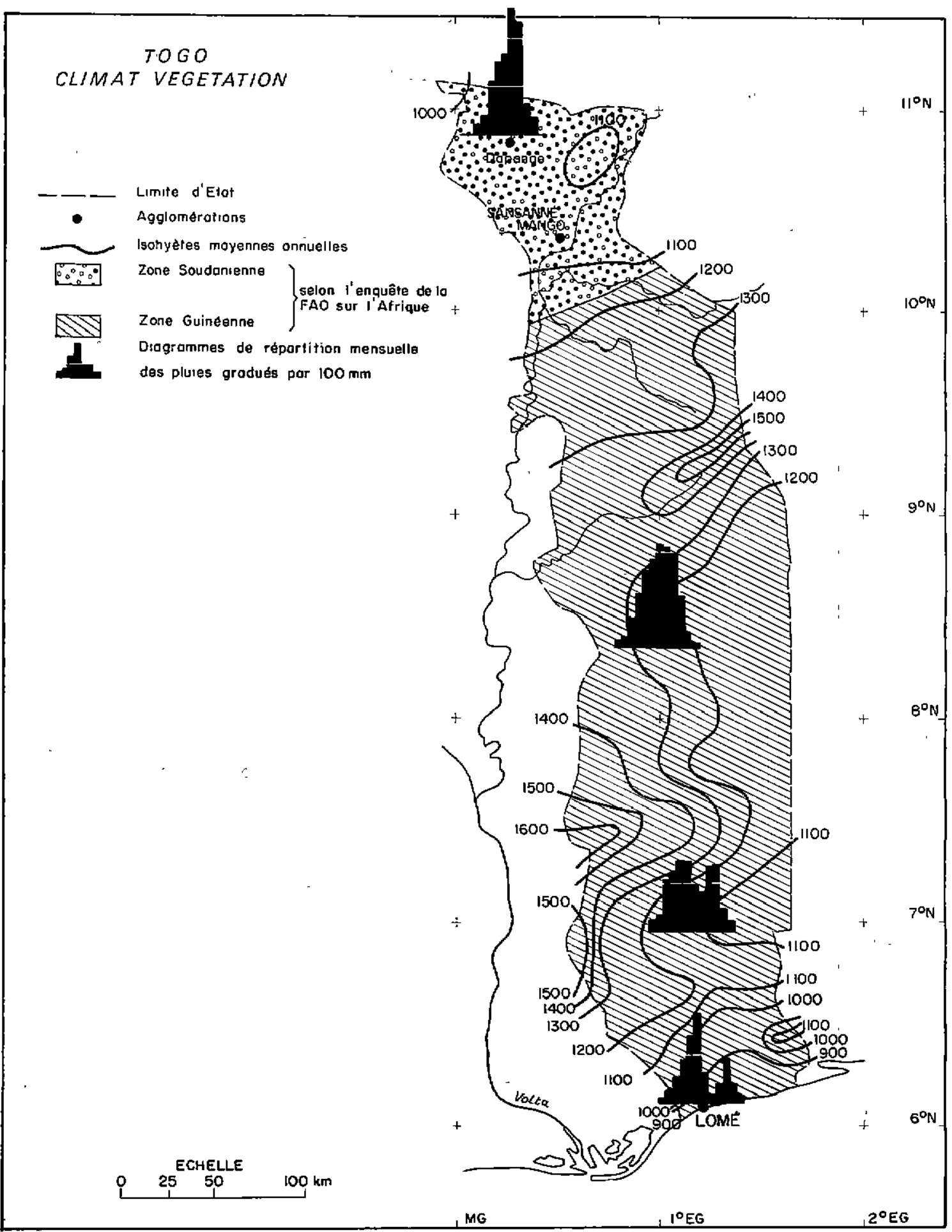

Carte II. - République du Togo - Clımal el végétation. 
comme des rivières permanentes. Les autres rivières sont à sec de décembre à mai, conservant des mares qui servent au ravitaillement des gens et du bétail, et permettent aux glossines de subsister pendant la saison sèche.

La plaine de Mandouri et la vallée moyenne de l'Oti sont inondées périodiquement en fin de scison des pluies.

\section{Végétation (carte II).}

La savane du Nord Togo présente des faciès très différents suivant la nature des sols et la densité de l'occupation humaine. Le boisement naturel à base de Khaya, Terminalıa, Anogeisșus, Pterocarpus, Borassus, Daniella, est très entamé par les cultures.

Les galeries forestières ont été très souvent abattues et ne subsistent que le long de certains cours d'eau ou portion de cours d'eau.

\section{Faune.}

La faune sauvage est peu abondante et n'est plus représentée que par des antilopes, quelques lions, des phacochères, quelques troupeaux de buffles et une dizaine d'éléphants parfaltement identifiés.

Le cheptel bovin est constitué essentiellement par des taurıns de race Borgou, plus ou moins métissés de zébu dans le Nord. L'effectif comprend environ 60.000 têtes, dont 40.000 dans la circonscription de Dapango et 20.000 dans la circonscription de Mango. La plupart des troupeaux sont confiés, par les propriétaires, à des Peuls. Ces troupeaux sont pratiquement sédentarisés, et n'effectuent que des déplacements très limités au cours des saisons.

Des troupeaux de boucherie, en provenance de Haute Volta ou du Dahomey, traversent cette région en suivant deux itinéraires principaux :

1. Nadjoundi ou Korbongou - Dapango Bogou - Mango.

2) Kondjoare ou Mandouri - Borgou Tchanaga - Mango.

A partir de Mango ces troupeaux sont dirigés soit vers le Ghana, soit vers Bassari ef le Sud du Togo.

\section{Population.}

La population de la Région des Savanes est très inégalement répartie, et il faut distınguer, tant au point de vue densité que répartition ethnique la circonscription de Dapango de la circonscription de Mango.

La densité de population dans la circonscription de Mango est en moyenne de 13 habitants au $\mathrm{km}^{2}$ et se situe parmi les plus faibles du pays. En revanche, dans la circonscription de Dapango, la densité mayenne atteint 35 habitants au $\mathrm{Km}^{2}$. Cette population est inégalement répartie, et l'on trouve des zones denses de plus de 100 habitants au $\mathrm{km}^{2}$ et des secteurs peu peuplés (moins de 10 habitants au $\mathrm{km}^{2}$ ). La population y est essentıellement composée de Moba et de Gourma.

Les Peuls, qui ne représentent que 4 p. 100 de la population totale, sont dispersés dans toute la circonscription à l'exception de la région SudOuest.

La majorité de cette population esł agricole. Les autres secteurs d'activité sont très peu représentés, et limités aux deux princıpaux centres.

\section{I. - RÉPARTITION GÉOGRAPHIQUE DES GLOSSINES}

\section{Historique.}

Les renseignements concernant la répartition et la systématique des insectes d'intérêt médical et vétérinaire au Togo sont très peu nombreux ef fragmentaires.

Il n'existe, à notre connaissance, aucune publication concernant la distribution des glossines au Togo. Les seuls renseignements que l'on possède sont :

a) une carte générale de distribution des tsétsés en Afrique publiée en 1953 par le Directorate of Surveys, de Londres, d'après des informations recueillies par POTTS.

b) une carte de répartition des glossines en Afrique Occidentale d'expression française, publiée en 1961 par l'O. R. S. T. O. M., et établie par RICKENBACH.

c) une série de cartes de distribution des espèces de glossınes, en Afrıque, publiées dans le bulletin de I'O. M. S. en 1963, et établies par FORD.

d) une liste des espèces de tsétsés, publiée dans le volume de HEGH : «Les Tsétsés», paru 
en 1929, et qui mentionne, pour le Togo, les espèces sulvantes :

Gl. palpalis, Gl. longipalpis, Gl. fusca, Gl.morsitons submorsitans, $\mathrm{Gl}$. tochinoides.

e) des points de capture cités, dans l'ouvrage de ZUMPT «Die Tsetsefliegen », paru en 1936, à propos des espèces suivantes :

Gl. fusca fusca, retrouvée jusqu'à Bismarckburg (50 km au Sud-Ouest de Blitta).

Gl. palpalis palpalis, fréquente partout jusqu'au 9o latitude Nord, puis sporadiquement en différents endroits jusqu'au $12^{\circ}$ latitude Nord.

Gl. tachinordes, largement répandue entre $8^{\circ}$ ef 130 de latitude Nord.

Gl. morsitans submorsitans, trouvée à Kete Kratchi (situé actuellement en territoire Ghanéen, sur la Volta).

Espèces actuellement présentes dans la région des Savanes.

Les séries de captures effectuées au cours des prospections n'ont révélé la présence que de deux espèces :

Glossina tochinoides West. et Glossina palpalis gambiensis Vand.

Il peut paraître assez surprenant que Gl. morsitans submorsitans, mouche de savane, présente en Haute-Volta, au Dahomey ef au Ghana, et signalée autrefois au Togo, n'ait pas été rencontrée au cours de ces prospections, d'autant que les caractéristiques climatiques de la région conviennent parfaitement aux besoins éthologiques de cette espèce.

En fait, il est vrassemblable que $G l$. marsitans occupait autrefois une grande partie de la région des Savanes.

A la suite du peuplement humain de plus en plus important, singulièrement dans la circonscription de Dapango, de la multiplication des surfaces cultivées, de la dissémination des villages, du déboisement et de la raréfaction du gibier, la mouche a été refoulée et a peu à peu disparu. Il est toutefois possible qu'il en subsiste quelques îlots qui n'ont pu être décelés, en raison des difficultés d'accès. Ce peut-être le cas pour la plaine de Mandouri.

L'enquête entomologique a été effectuée en septembre-octobre, époque où les zones d'inondation sont les plus étendues ef où l'humidité élevée permet aux glossines d'occuper des habitats beaucoup plus nombreux qu'en saison sèche.

II est donc probable que les aires respectives des deux espèces, telles qu'elles ont pu être établies au cours des prospections, sont beaucoup plus réduites en saison sèche. Dans les cours d'eau non permanents, Gl: polpolis sera absente pendant la salson sèche, et seule pourra subsister Gl. tochinordes là où la végétation et la présence de mares entretiendront un microclimat compatible avec ses besoins.

En saison des pluies, Gl. palpalis ne dépasse pas $10^{\circ} 50^{\prime}$ de latıtude Nord, ce qui constitue la lımife extrême son arre d'extension 'maximum (carte III).

Les points de capture suivants précisent cette limite :

- Rivière Kpong, près du village Sissiaga.

- Rivière Mog-Bong, près de Sounsouri.

- Rivière Pambonou, au sud du village Gouani, vers la fosse aux Lions.

- Rivière Okougou, près du village Ogaro, route de Sawaga à Borgou.

- Rivière Sansargou, au gué de Borgou.

Gl. polpolis a presque toujours été trouvée coexistant aves Gl. tachinoides, et généralement en nombre plus faible.

Gl. tachinoides a été capturée beaucoup plus au Nord (Rivière Sansargou, près du village Ponio), et a été retrouvée dans toute l'étendue de la zone de prospection, ce qui est parfaitement en accord avec son aire de répartition générale, qui atteint le $13^{\mathrm{e}}$ degré de latıtude Nord.

\section{A. - Secleur de Sansanné-Mango.}

Ce secteur est arrosé par deux importants cours d'eau : l'Oti et la Koumangou.

L'Oti coupe en diagonale le secteur de Mango, depuis la frontıère avec le Dahomey, à $50 \mathrm{~km}$ au Nord-Est de Mango, jusqu'à la frontière avec le Ghana, à $14 \mathrm{~km}$ au Sud-Ouest de Mango.

C'est une rivière large, qui serpente dans une plaine peu accidentée. D'anciens méandres, coupés du lit principal, constituent des mares permanentes. L'Oti est bordée, dans la majeure partie de son cours, par de larges zones d'inondations, et, de place en place, par des galeries forestières plus ou moins importantes. 
Glossina tachinordes est l'espèce la plus fréquente. Elle se rencontre tout le long du cours d'eau, et suit les zones d'inondation. Ses gîtes, en saison sèche, se trouvent dans les galeries forestières, à partir desquelles elle envahit, en saison des pluies, toute la zone d'inondation. C'est ainsi qu'elle a été capturée dans des plaines inondées, à la hauteur du village Manion (S. E. de Mango), à l'ancien bac au Sud de Mango, dans Mango même, au domaine Gravillou, et à la hauteur de Tchanaga.

Glossina palpalis gambiensis est beaucoup moins fréquente, et n'a été capturée qu'aux environs de Tchanaga, à l'occasion d'une remontée de l'Oti en pirogue. A cet endroit, le cours de l'Oti fait deux coudes successifs, dont les rives sont bordées par des galeries forestières et des îlots forestiers importants servant de gîtes à cette espèce.

Le Koimepouarbaga, affluent rive droite de l'Oti, prend ses sources dans les collines situées autour de Bidjanga, puis coule d'Ouest en Est en passant par Nagbeni. A $15 \mathrm{~km}$ au Nord de Tchanaga, son cours s'infléchit vers le Sud. II se jette dans l'Oti à la hauteur de Tchanaga.

Aux environs de Bidjanga, les différents cours d'eau (Dapon, Kpeidjak), qui se réunissent pour former le cours principal, coulent dans une région de savane arbustive claire où les zones cultivées sont nombreuses. Les rives de ces affluents sont dénudées, ef les glossines en sont absentes.

A partir du village Kpinbonga, la galerie forestière fait son apparition, et se retrouve, plus ou moins importante, tout le long du cours d'eau. La forêt est particulièrement dense à l'Est de Nagbeni.

Gl. tachinoides et Gl. palpalis gambiensis ont été capturées à Kpinbonga, Nagbeni, Biaga, en abondance. A partir de Kpinbonga, et jusqu'à I'Oti, les glossines sont partout présentes. II faut noter qu'il existe un important foyer de trypanosomiase humaine autour de Nagbeni.

Le. Koukombou, autre affluent rive drolte de I'Oti, fait frontière avec le Ghana, dans la portion de son cours comprise entre la forêt de Galangachi et le confluent avec l'Otı. C'est un cours d'eau important, qui traverse une zone inondée en saison des pluies. II est bordé de rideaux d'arbres servant de gîtes à Gl. tachinoides.
La Koumangou important affluent rive gauche de l'Oti, coule d'Est en Ouest, en suivant à peu près le parallèle $10^{\circ} 10^{\prime}$. Depuis la forêt de la Kéran, jusqu'à la longitude $0^{\circ} 35^{\prime}$, le cours de cette rivière est bordé de grosses galeries forestières qui abritent en abondance Gl. tachinoides et Gl. palpalis gambiensis.

Au-delà du méridıen $0^{\circ} 35^{\prime}$, la forêł galerie disparaît et le cours d'ecu serpente dans une plaine ınondable. Dans cette régıon, il n'a été capturé aucune glossine.

Sur les affluents rive gauche de la Koumangou, aucune glossine n'a également pu être décelée. La plupart de ces affluents sont de petits cours d'eau temporaires aux rives dénudées, qui traversent d'importantes zones de culture. II est très vraisemblable que les glossines en solent absentes.

Quelques-uns des affluents rive droite de la Koumangou ont pu être prospectés, en particulier le Yaweni, vers le village Paio, le Wapoti, vers le village Sigbinga ; un petit affluent du Foleboula, près de Gando Namoni. Sur ces trois cours d'eau, seule Gl. tachinoides a pu être capturée, en plus ou moins grande abondance.

\section{B. - Secteur de Dapango.}

Le réseau hydrographique de ce secteur comprend essentiellement la Koulougona, le Yamboul, la Sansargou ef son principal affluent, le Bamoina.

Lo Koulougona est une rivière permanente qui court d'Est en Ouest, depuis la forêt de la Fosse aux Lions, où elle prend le nom de Mog-Bong, jusqu'à la frontière du Ghana, où elle se jette dans la Biankourı. Cette rivière traverse une zone inondée en saison des pluies, et ne comporte que quelques galeries forestières situées d'une part en aval de la forêt de la Fosse aux Lions, dans la région de Sounsouri ; d'autre part, à l'Est de Nano, en deux ou trois points. Ces galeries forestières, sont infestées par Gl. palpalis gambiensis.

Autour de Nano, les rives sont dénudées, et il n'a été capturé aucune glossine.

Dans la forêt de la Fosse aux Lions, la Napabour, affluent du Mog-Bong, est infestée par Gl. tochinoides, qui y est très abondante et agressive: 


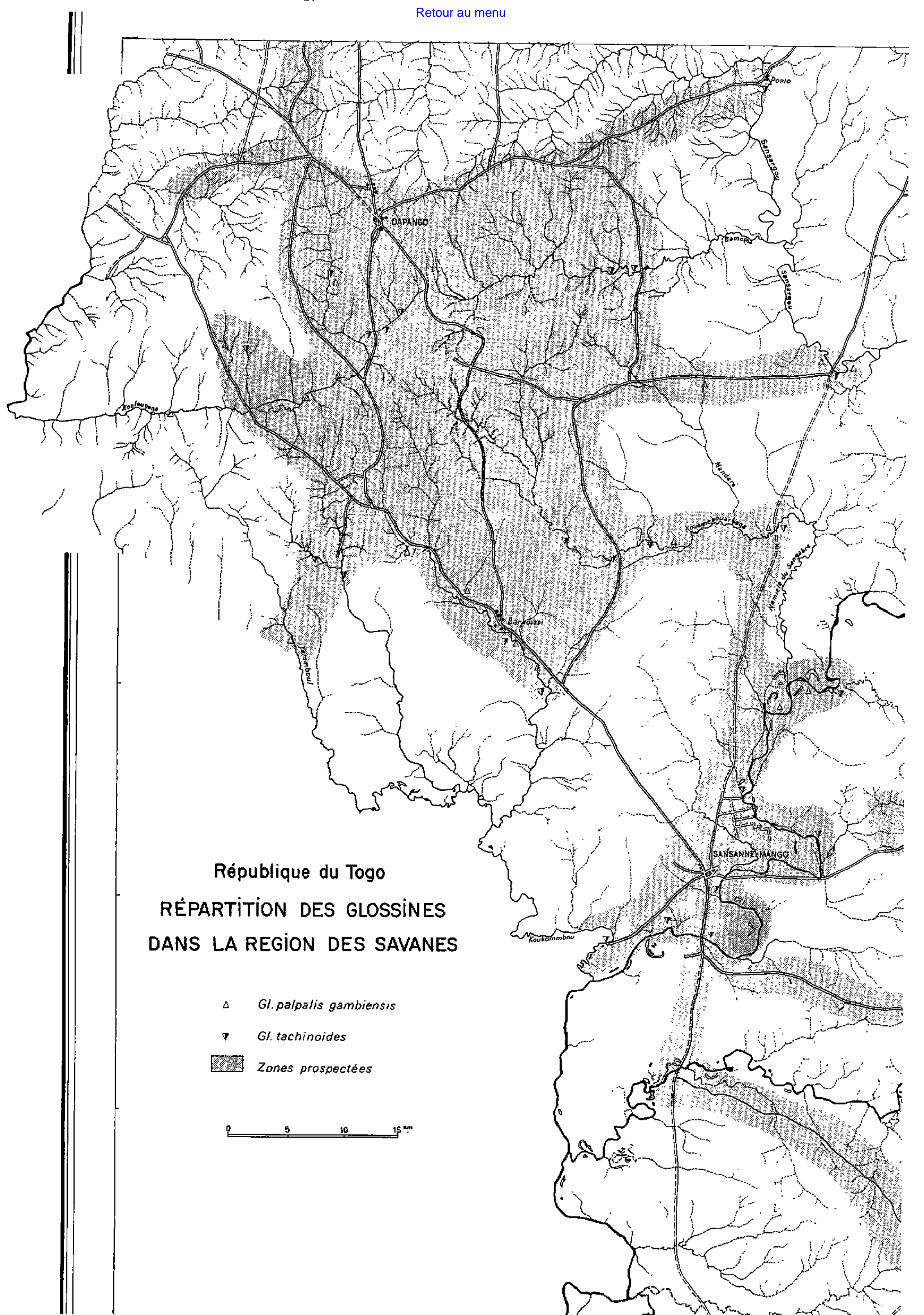


Retour au menu

at

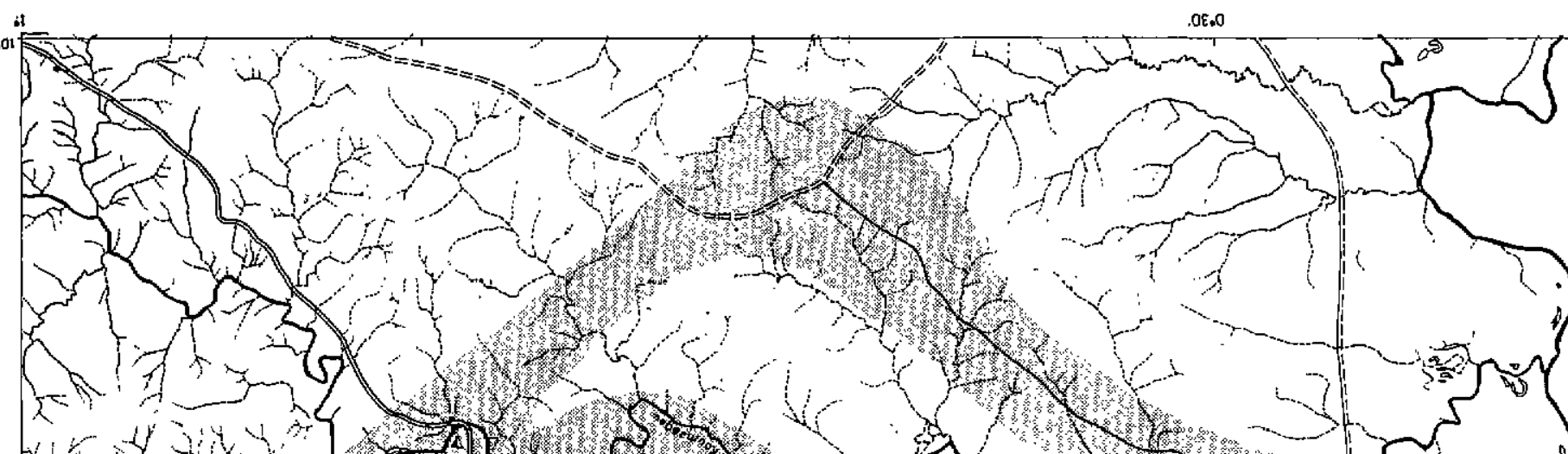
(1) 
Sur la rivière Kpong, affluent rive droite du Mog-Bong, aux environs des villages Nakpabak et Sissiaga, Gl. tochinoides et Gl. palpalis gambiensis sonf présentes en faible quantıté.

Le Yamboul, qui prend source aux monts Boureleman, à $8 \mathrm{~km}$ environ à l'Est de Nano, descend vers le Sud et forme, à partir du village Yamboul, la frontière avec le Ghana. Sous le nom de Naba, il rejoint la Koukombou à l'extrémité Sud de la forêt classée de Galangachi. Ce cours d'eau reçoit deux affluents principaux : le Kountoné qui prend le nom de Wandégué avant de rejoindre la Naba; le Laktaon, qui longe, à partir de Natigou, la route de Dapango à Mango, et, après avoir traversé Barkoissi, s'infléchit vers le Sud, Sud-Ouest pour rejoindre, sous le nom de Gambara, la Naba.

Le Yamboul, aux environs du village du même nom, esł bordé par une galerie forestière de faible importance, qui abrite quelques $G l$. palpalis gambiensis.

Son affluent, le Kountone, héberge, vers le village Bantierk, Gl. tachinoides.

Sur le Laktaon la forêt galerie débute à environ 6 d̀ $7 \mathrm{~km}$ en amont de Barkaissi, puis devient de plus en plus importante jusqu'au Sud du village Poloti. Cette galerie forestière abrite, en abondance, à la fois Gl. tochinoides et Gl. palpalis gambiensis. Cette dernière espèce est partıculièrement abondante ou niveau de la station d'Elevage de Barkoissi. Ses gîtes se situent dans toute la galerie forestière bordant le cours d'eau entre Barkoissi et Poloti. Les petits affluents situés en amont de Barkoissi (rivière Koubangou, rivière Koukoulou) hébergent également les deux espèces.

La Sansargou est un important cours d'eau qui coule du Nord au Sud depuis Ponio jusqu'à Borgou. A partir de Borgou il a une direction Ouest-Est ef rejoint l'Oti sous le nom de Kanboanga. Son principal affluent, la Bamoina, coule d'Ouest en Est en passant par Kankanpieni.

Autour de Ponio, la Sansargou, qui forme frontière avec la Haute-Volta, coule dans un lit encaissé, aux rives dénudées. Les glossines en sont absentes, mais ont été capturées sur les petits affluents de sa rive droite, qui conservent sur une centaine de mètres, des restes de galerie forestière, servant d'abri à Gl. tachinoides.

A partir du confluent avec la Bamoina, on retrouve des îlots de forêt galerie qui s'étendent jusqu'à proximité de Borgou et hébergent à la fois Gl. tachinoides et Gl. palpolis gambiensis.

La Boimana a été prospectée autour de Gallé et de Nataré.

A Gallé, les rives sont totalement dénudées, les zones cultivées sont nombreuses, et il n'a pas été trouvé de glossine.

Près de Nataré, la rivière est bordée par une végétation basse et buissonneuse qui abrite Gl. tachinoides.

\section{II. - TABANIDAE}

Au cours des prospections, plusieurs insectes d'intérêt vétérinaire ont été également capturés. II s'agit essentiellement de Tabonidae, très abondanis à l'époque où a été effectuée l'enquête.

Les espèces suivantes ont été capturées :

Genre Haematopota Meigen, 1803.

H. exiguicarnuta, capturé dans la forêt de la Fosse aux Lions et le long de la rivière Kariata.

$H$. hostata, capturé le long de la rivière MogBong.

$H$. bullatifrons, capturé sur la rivière Kariata.

H. pallidipennis, capturé au Nord de Timbou, à la frontière de Haute-Volta.

Genre Ancala Enderlein, 1922.

A. latipes, capturé le long de la rivière MogBong.

A. fasciota, capturé sur la rivière Mog-Bong et près de Borgou.

Genre Atylotus Osten-Sacken, 1876.

A. fuscipes, trouvé sur les rivières Kariata, Kountoné, Sansargou, ainsi qu'à Dapango, Nassablé, Ponıo, Barkoissi, Timbou.

A. agrestis, trouvé le long de la Sansargou, près de Ponio.

Genre Tabonus Linné, 1758.

T. gratus, capturé à Dapango, Nassablé, Barkoissi, Palo.

T. biguttatus, trouvé à Gando-Namoni, Mango, Timbou, Ponio. Les deux variétés (variété jaune et variété grise) sont présentes dans ces régions.

$T$. par, capturé près de Borgou et aux environs de Bidjanga.

T. quadrisignatus, trouvé le long des rivières Mog-Bong et Kariata. 
T. taeniola, espèce largement répandue, capturée sur la Kariata, la Koumangou, à Mango, a Tchanoga, près de Borgou, à Dapango, à Nassablé, à Ponio.

$T$. sugens, trouvé le long de la Kariata.

T. nyasoe (?), capturé près de Kountouri, à Dapango et au Nord de Timbou.

Genre Chrysops Meigen, 1800.

C. distinctipennis, trouvé près de Kariata.

C. longicornis, capturé à Dapango.

\section{III. - INFLUENCE DE LA RÉPARTITION \\ DES GLOSSINES SUR LES TRYPANOSOMIASES HUMAINES ET ANIMALES}

\section{A. - Trypanosomiase humaine.}

La trypanosomiase humaine sévit à l'état' endémique dans la circonscription de Mango, où il existe un important foyer, le long de la Koimepouarbaga, autour des viliages Kpinbonga, Nagbeni et Biaga.

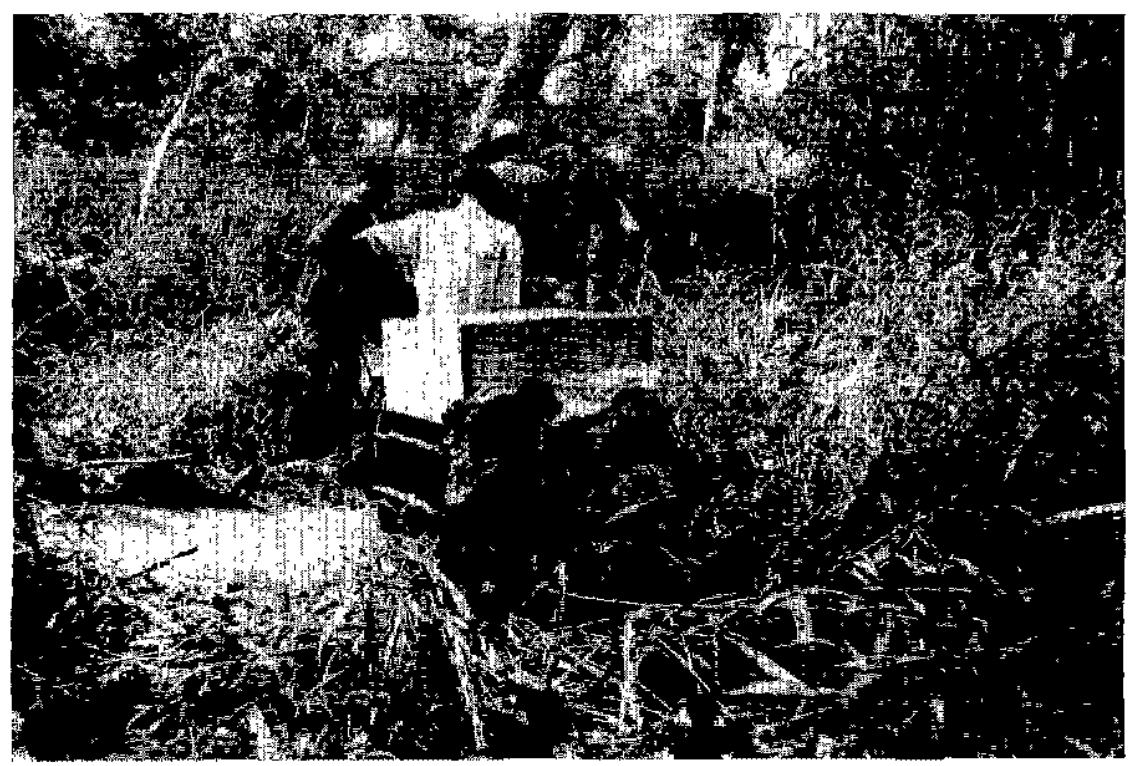

Fig. 1. - Rivière Koimepouarboga - Au premier plan, piège c̀ glossine installé par le Service de Santé.

Les malades viennent se faire soigner à I'hôpital de Mango. L.'hôpital de Dapango a également un certain nombre de trypanosomes en traitement, originaires de la région de Nakitindi Laré, près de Sawaga (Noki Est). II est probable que les malades qui se font soigner à Dapango se sont infestés dans la région de Nagbeni, ou sur la rivière Mandaré.

D'après les renseıgnements fournis par l'Hôpital de Mango, il existait au 1er janvier 1967, 73 trypanosomés en traitement. Entre le 1er janvier 1967 et le 30 septembre 1967, il a été dépisté 43 nouveaux malades.

\section{B. - Trypanosomiases animales.}

La présence des glossines dans une grande partie de la Région des Savanes influe sur la répartition des grands animaux domestiques de façon très significative. C'est ainsi que l'élevage des chevaux et des anes est limité à la partie située au Nord de Dapango, vers la frontière de Haute-Volta.

De même, l'élevage du Zébu et des métis de Zébu ne s'étend pas au sud du parallèle de Dopango.

Partout ailleurs, l'élevage bovin est constitué par des taurins de race Borgou, animaux de petit format, proches du bétail de race Baoulé ou lagune, mais de robe généralement fauve brûlé. Ces animaux sont naturellement trypanotolérants, et sont répartis en petits troupeaux dans toute la Région des Savanes, jusqu'au Sud de Mango.

La répartition de ces troupeaux est assez 


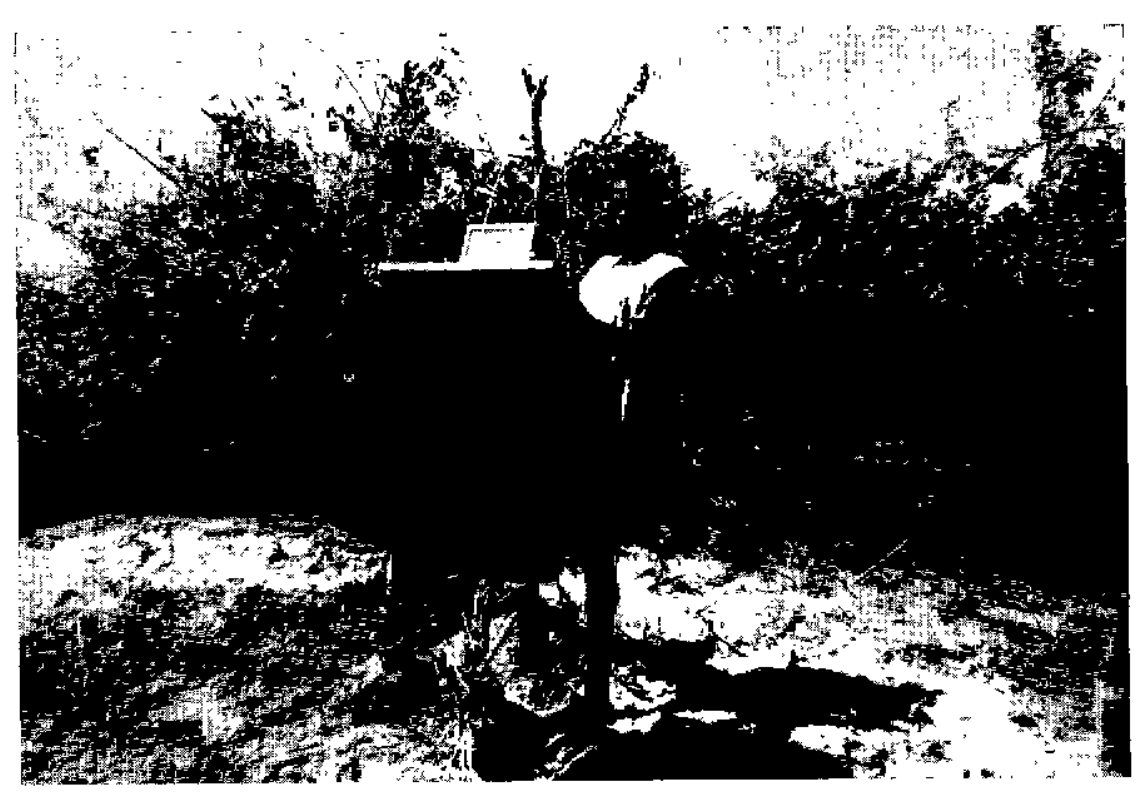

Fig. 2. - Rivière Sansargou, gué de Borgou - Piège à Glossine type Lewillon.

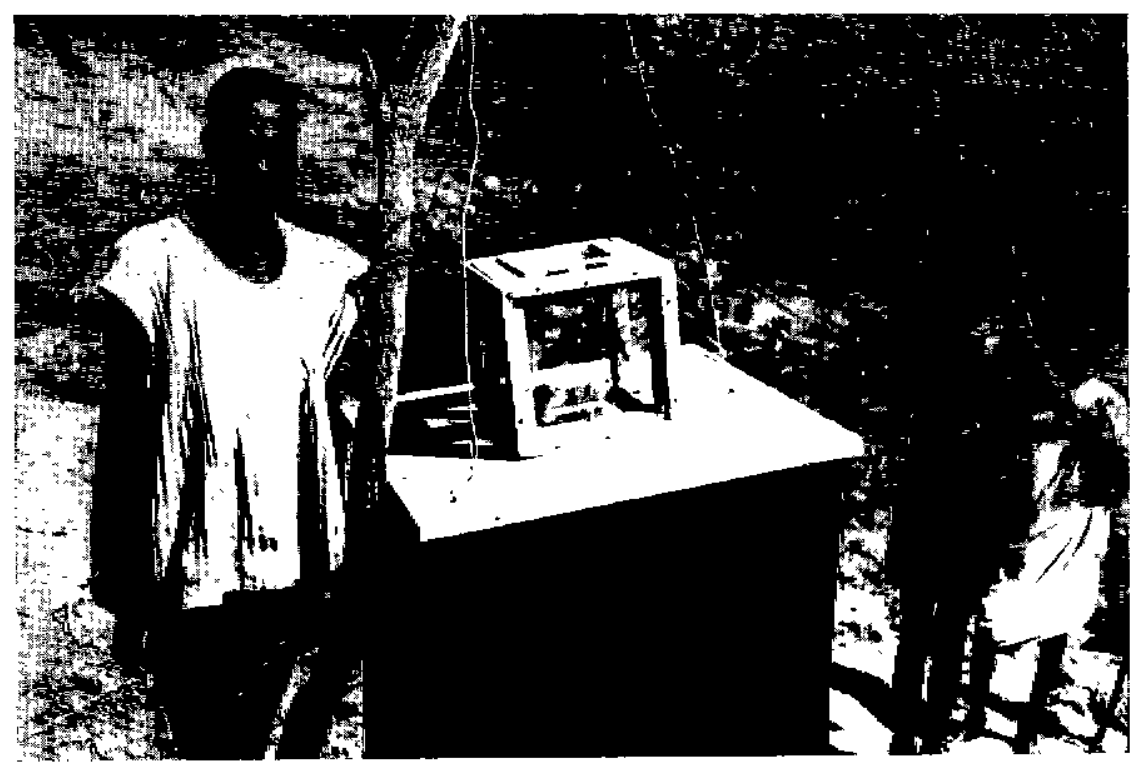

Fig. 3. - Piège à Glossine type Lewillon - détail. 
inégale. Le. plus grand nombre est situé dens.la. circonscription de Dapango, dans les Secteurs Nord, Nord-Ouest, Sud-Ouest et Est, soit dans un rayon d'une trentaine de kilomètres autour de Dapango.

Il existe en outre un troupecu près de Ponio, et deux ou trois troupeaux dans la région de Mandour - Borgou.

Dans la circonscription de Mango, les troupeaux sont situés soit dans la vallée de l'Oti, autour de Niamélé, Tchanaga, Koundjouaré, Koussigou et Mogou, solt dans la vallée de la Koumongou, autour de Baoulé, Sigbınga, Panga, Kountouri. Il existe également des troupeaux autour de Takpammba, dans la vallée de la Kara.

En général, les cas de trypanosomiase sur ce bétail trypanotolérant, sont relativement peu nombreux, bien qu'il soit difficile de se farre une opinion précise en la matière, les seuls cas recensés étant ceux signalés par les éleveurs. VAUCEL (1963) rapporte qu'une enquête sur le parasıtisme, portant sur 1502 échantillons, a décelé 3 p. 100 de porteurs de trypanosomes. $T$. vivax, $T$. congolense et $T$. brucei sont les espèces signalées.

\section{IV. - CONCLUSIONS}

L'étude de la répartition des Glossines dans la Régıon des Savanes montre que celles-ci sont présentes le long des cours d'eau permanents bordés par des galeries forestières. On les trouve pratiquement sur tout le réseau hydro- graphique, dès l'instant: que celui-ci présente une végétation arborée suffisante pour créer le micro-climat qui leur est indispensable.

C'est le cas, essentiellement, pour les grands cours d'eau tels que :

- L'Oti et ses principaux affluents (Sansargou, Koimepouarbaga, Yamboul ef Koukommbou avec le Laktoon).

- La Koumangou.

Toutefois, l'action humaine a eu une influence considérable sur cette répartition, en détruisant les galeries forestières de nombreuses rivières, et en étendant les zones de culture. C'est ainsi que, au Nord de Dapango, les glassines sont pratiquement absentes de la plupart des cours d'eau.

Il est enfin très important de noter l'absence de l'un des plus importants vecteurs des trypanosomiases animales : Glossina morsitans, espèce moins exigeante que $G$ l. tachinoides et Gl. palpalis, et se contentant de régions plus sèches, d'une végétation moins dense et d'une eau plus rare.

De ce fait, l'élevage bovin est possible dans les savanes comprises entre les cours d'eau, si l'on prend soin d'éviter la proximité des galeries forestières.

Institut d'Elevoge et de Médecine Vétérinaire des Pays Tropicaux. Laboratoire d'Entomologie Maisons-Alfort.

\section{SUMMARY}

\section{An entomological survey of the Savanna region in the Togo Republic}

The distribution of tsetse flies in the Savanna region is characterised by :

1. - Absence of Gl. morsitons, at least within the limits of the area studıed.

2. - Presence of Gl. tochinoides and Gl. polpolis gombiensis, which frequently co-exist.

These two species are found in places of dense overhead vegetation but always in close praximity to permanent water. Gl, tochinoides is the dominant species,

Owing to the absence of Gl. morsitans, cattle rearing is possible in the savannas between the rivers.

A list is also given of the species of Tabanidae caught during the survey. 


\section{RESUMEN}

Encuesta entomalogica en la región de las sabanas (República de Togo)

En la región de las sabanas, se carocteriza la repartición de las glosinas por:

1. - Ausencia de Gl. morsitons, en lo concerniente a las zonas observadas.

2. - Presencia de Gl. tachinoides y de Gl. palpolis gambiensis, que frecuentemente coexisten,

Se encuentran las dos especies en una vegefación de tipo galeria, pero establecidas cerca de extensiones de agua permanentes. Gl. tachinoides es la especie más numerosa.

La ausencia de Gl. morsitans permite la ganaderia de los bovinos en las sabanas situadas entre los ríos.

Se enumeran las especies de Tabonidae apresadas durante ésta encuesta.

\section{BIBLIOGRAPHIE}

HEGH (E.). - Les Tsétsés. Tomie I, Bruxelles, 1929.

FORD $\left\{J_{3}\right\}_{\text {. }}$ - The distribution of the vectors of African pathogenic trypanosomes. Bull. Org. mond. Santé, 1963, $28: 653 m 669$.

RICKENBACH (A.). - Carle de répartition des glossines en Afrique occidentale d'expression française. O. R. S. T. O. M., 1961. VAUCEL (M. A.), WADDY (B. B.), de ANDRA-
DE DA SILVA (M. A.) ef PONS (V. E.). Répartition de la trypanosomiase africaine chez I"homme et les animaux. Bull. Org. mond. Santé, 1963. 28 : 545-594.

ZUMPT (F.). - Die Tsetsefliegen, Jena, G. Fischer, 1936.

Distribution of Tsetse species in Africa. Directorate of Surveys. London, Edward Stanford Ltd, 1953. 